\title{
Satisfacción con la atención en urgencias pediátricas: influencia del funcionamiento familiar y la alteración emocional
}

\author{
Satisfaction with care received in pediatric emergency services: \\ impact of family interaction and emotional disturbance
}

Antonio Fernández-Castillo ${ }^{1}$

María J. Vílchez-Lara ${ }^{2}$

${ }^{1}$ Departamento de Psicología Evolutiva y de la Educación,

Universidad de Granada. Campus de Cartuja $S / n^{\circ}$ 18071 Granada España. afcastil@ugr.es

${ }^{2}$ Hospital Universitario

"Virgen de las Nieves",

Servicio Andaluz de Salud.

Granada España.

\begin{abstract}
This study addresses the relationship between two key elements in satisfaction with pediatric emergency services, namely emotional disturbance and family interaction. The main goal is to determine whether family cohesion and adaptability and anger may be associated with differential levels of satisfaction among parents whose children were attended in pediatric emergency units. It is a descriptive study for which a sample of 711 parents whose children were attended in 6 pediatric emergency services was studied. The Spanish version of the State-Trait Anger Expression Inventory-2 (Staxi-2), the Satisfaction with Healthcare Services Scale and the Family Adaptability and Cohesion Evaluation Scale (Faces) were used respectively. Data analysis included a Spearman bivariate correlations analysis, Kruskal Wallis, Mann-Whitney test and path-analysis using a structural equations model. The results support the hypothesis that higher levels of family cohesion and adaptability as well as lower levels of anger are associated with higher levels of satisfaction among parents.
\end{abstract}

Key words Patient satisfaction, Anger, Family relations, Emergencies, Emotional disturbance
Resumen Este estudio aborda la relación entre dos elementos clave en la satisfacción con los servicios sanitarios de urgencias pediátricas: la afectación emocional y el funcionamiento familiar. El objetivo general es determinar si la cohesión, la adaptabilidad familiar y la ira pueden asociarse a niveles diferenciales de satisfacción en progenitores cuyos hijos eran atendidos en urgencias pediátricas. Se trata de un estudio descriptivo transversal para el que se trabajó con una muestra de 711 progenitores que habian sido atendidos en 6 servicios de urgencias pediátricas. Se utilizaron respectivamente las versiones españolas del Inventado de Expresión de Ira Estado-Rasgo-2 (STAXI-2), la Escala de Satisfacción con los Servicios Sanitarios y la Escala de Evaluación de la Cohesión y Adaptabilidad Familiar (Faces). El análisis de datos incluyó un análisis de correlaciones bivariadas de Spearman, pruebas de Kruskal Wallis, pruebas de Mann-Whitney y un path-analysis mediante un modelo de ecuaciones estructurales. Los resultados soportan la idea de que mayores niveles de cohesión y adaptabilidad familiar así como menores niveles de ira, se asocian a mayores niveles de satisfacción en los padres. Nuestros datos no reflejan diferencias significativas en ira entre padres $y$ madres.

Palabras clave Satisfacción del paciente, Ira, Relaciones familiares, Urgencias, Alteración emocional 


\section{Introducción}

La satisfacción con la atención recibida en diversos servicios sanitarios en general y en urgencias en particular, ha sido objeto de estudio desde hace tiempo tanto por sus implicaciones en el bienestar de los pacientes, como por su papel como indicador de funcionamiento, eficiencia y calidad de esos servicios ${ }^{1-3}$.

Entender la satisfacción exclusivamente como la cumplimentación de las necesidades del usuario, sería una aproximación demasiado simplista teniendo en cuenta que se trata de una variable compleja, de difícil conceptualización y más aún en contextos sanitarios ${ }^{4}$. En su delimitación conceptual influyen diversos aspectos y factores, que además, suelen interactuar de forma compleja y variable entre sí. De hecho, en su definición han de tenerse en cuenta aspectos tales como que al usuario del sistema de salud se le preste la atención que necesita en el momento preciso y que sea convincente según lo que demanda y espera tanto él, como los profesionales del propio sistema ${ }^{5}$. Y por si todo esto fuese poco, en el fondo estamos ante una percepción subjetiva del usuario, que puede no coincidir con el punto de vista de los profesionales, y que en todo caso variará a lo largo del tiempo, entre situaciones y entre sujetos, manifestando oscilaciones adicionales en función de variables tales como el nivel educativo y socioeconómico, el género y la edad $\mathrm{u}$ otras como las experiencias previas, el apoyo familiar durante el proceso de atención sanitaria, la presencia de alteraciones emocionales durante la estancia, ciertas opiniones y creencias de los usuarios de salud, etc. ${ }^{6-8}$.

Frecuentemente y por su influencia en el proceso de atención sanitaria, se ha diferenciado entre cuatro bloques básicos en los que se basa la satisfacción tanto del usuario como de sus familiares en contextos hospitalarios y de salud. En primer lugar se otorga importancia crucial a la satisfacción con la atención recibida del personal sanitario, en segundo lugar estaría la atención recibida del personal de apoyo, en tercer lugar figurarían las condiciones físicas, ambientales y de confort y en un cuarto grupo se incluirían variables relativas al acceso y funcionamiento del servicio específico ${ }^{3}$.

En el caso de la atención sanitaria pediátrica y especialmente en la atención de urgencias pediátricas, la dificultad de evaluación de la satisfacción es añadida si tenemos en cuenta que frecuentemente se parte de la opinión de los progenitores y las familias atendidas en tales servi- cios, y que las variables aludidas anteriormente interactúan entre sí de forma diferente cuando son expresadas en relación con un hijo ${ }^{9,10}$.

Parece haber consenso sobre que la atención sanitaria en contextos de urgencias pediátricas presenta particularidades que la hacen especial. Entre ellas han sido tenidas en cuenta una mayor alteración emocional tanto de familiares como de los pacientes, tiempos de espera que suelen percibirse por los usuarios como elevados, una atención recibida que se percibe como breve o aspectos como la comunicación con el personal. Todos estos aspectos específicos podrían hacernos esperar que la satisfacción fuese menor en estos ámbitos. Sin embargo, no se han encontrado diferencias en satisfacción parental con la atención pediátrica recibida por los hijos cuando se comparan servicios de atención primaria con urgencias pediátricas pero sí que la creencia en la existencia de un abuso en la prescripción de fármacos en atención pediátrica se asocia a menor satisfacción en los padres ${ }^{11,12}$. En relación con la alteración emocional, parece contrastado suficientemente que la presencia de ansiedad, estrés o ira, entre otras variables, suele estar presente durante la atención pediátrica ${ }^{6,13}$. Siendo estas alteraciones emocionales y específicamente la ira, variables asociadas a mayor insatisfacción en pacientes y familiares, tanto en servicios generales de urgencias como en servicios pediátricos ${ }^{14,15}$. En esta línea, estudios recientes han encontrado que la dificultad en el acceso a los servicios sanitarios, los tiempos de espera elevados, la información deficitaria o no comprendida, o la mayor gravedad percibida por los progenitores en sus hijos, son variables asociadas a mayores niveles de alteración emocional y en concreto a ira ${ }^{16-18}$.

En relación con la ira, ésta ha sido en diversas ocasiones conceptualizada a lo largo de una estructura tridimensional compuesta por sus expresiones verbal, física y como sentimiento ${ }^{19,20}$. Mientras que la dimensión verbal se relaciona con expresiones lingüísticas y comunicativas de irritación, cólera, etc., el componente físico hace referencia a expresiones motoras y la ira como sentimiento hace referencia a emociones y sentimientos de indignación, enfado, etc. Estas tres dimensiones suelen entenderse como los componentes fundamentales de una reacción de alteración emocional transitoria a situaciones de frustración o percibidas como amenazantes, que es como en ocasiones se define la ira y que se agrupan por otros autores bajo la división de expresiones externas e internas ${ }^{20}$. En relación con posibles diferencias de género, y con la excepción de 
la dimensión de expresión física de la ira, donde los hombres han alcanzado niveles significativamente mayores, lo habitual ha sido una tendencia a la inexistencia de diferencias estadísticas entre hombres y mujeres en los niveles generales de ira como estado, en la expresión como sentimiento y en la dimensión verbal ${ }^{20}$.

Por lo que respecta al contexto sanitario pediátrico y específicamente en urgencias pediátricas, la alteración emocional y la ira suelen estar presentes, siendo además la ira como sentimiento mayor en urgencias que en otros servicios de atención pediátrica ${ }^{21-23}$.

El estudio del funcionamiento de las alteraciones emocionales en contextos de atención sanitaria pediátrica ha incluido desde hace tiempo las relaciones familiares, habiéndose encontrado por ejemplo que el contagio emocional entre padres e hijos es algo frecuente por sus implicaciones contextuales o que aspectos tales como el funcionamiento de las familias en estos ámbitos predice diferencias significativas en los niveles de ansiedad y otras alteraciones emocionales ${ }^{16}$. Entre estos aspectos del funcionamiento familiar, los más relevantes parecen ser la cohesión familiar, la comunicación o la capacidad de adaptación en esa situación, variables estas que podrían jugar un papel importante en el ajuste psicológico y en el afrontamiento a estímulos y situaciones generadoras de alteración emocional en contextos sanitarios.

La cohesión en el seno de una familia ha sido definida como el grado de apego emocional que sus miembros establecen y mantienen entre sí. Incluye además aspectos funcionales tales como la cooperación entre los miembros, las expresiones de afecto, respeto, la cercanía, o la capacidad de apoyo, ayuda, etc. ${ }^{24}$. Paralelamente, la adaptabilidad, capacidad de adaptación o flexibilidad ha sido entendida como la capacidad de llevar a cabo cambios en el entorno familiar y su funcionamiento, en función de que una situación los requiera. Se relaciona esta variable con aspectos como la flexibilidad de rol, el liderazgo, el control y la disciplina entre sus miembros y determinados estilos y hábitos comportamentales, entre otros aspectos ${ }^{25}$. Por último suele incluirse en las dinámicas de funcionamiento familiar el análisis de los procesos y habilidades de comunicación por cuanto serían un aspecto inherente y que permite el óptimo desarrollo de la cohesión y la adaptación familiar, así como la relación entre ellas ${ }^{25}$.

$\mathrm{Y}$ aunque no muchos estudios han relacionado el funcionamiento familiar con los niveles de ira y otras alteraciones emocionales durante la atención en urgencias pediátricas, lo que sí parece claro es que un nivel de cohesión familiar o de funcionamiento familiar adecuado parece asociarse a la reducción de alteración emocional y a menores niveles de ansiedad en otros contextos ${ }^{16,24}$. Tampoco existen muchos estudios sobre la posible influencia del funcionamiento familiar y la presencia de ira en relación con la satisfacción con la atención recibida en contextos de urgencias pediátricas.

Por todo ello, los objetivos que nos planteamos en nuestra investigación, son en primer lugar determinar una posible asociación entre las diferentes dimensiones de ira consideradas, el funcionamiento familiar y la satisfacción en una muestra de padres cuyos hijos estaban siendo atendidos en urgencias pediátricas. Según nuestras referencias, esperamos encontrar a) que unos niveles adecuados de funcionamiento familiar, (cohesión y adaptabilidad familiar) se asocien con mayores niveles de satisfacción con la atención recibida en urgencias y b) con menores niveles de ira en las tres dimensiones consideradas (sentimiento, verbal y motora). Además esperamos igualmente que menores niveles de ira en esas manifestaciones, se asocien a mayores niveles generales de satisfacción.

En segundo lugar, nos planteamos como objetivo profundizar en la asociación anterior y sondear posibles diferencias en los niveles de ira en función de los niveles de cohesión y la adaptabilidad familiar así como en satisfacción en función del funcionamiento familiar y los niveles de ira. Esperamos encontrar, a tenor de la bibliografía consultada, que los niveles de ira serán menores conforme mayores sean los niveles de cohesión y adaptabilidad familiar, así como que los niveles de satisfacción expresada serán mayores conforme menores sean los niveles de ira en los padres y mayores sean los niveles de cohesión y adaptabilidad familiar.

El tercero de nuestros objetivos pretende analizar la existencia de posibles diferencias en ira en general entre hombres y mujeres en este contexto. Nuestra hipótesis de partida nos hace esperar que no haya diferencias significativas tanto en el nivel general, como en las diferentes dimensiones de ira consideradas, entre hombres y mujeres a tenor de la bibliografía consultada, a excepción de la dimensión de ira en su expresión física, donde los padres podrían alcanzar niveles superiores a las madres.

Por último el cuarto de nuestros objetivos se centra en estudiar si el funcionamiento familiar 
puede asociarse a niveles diferenciales de satisfacción, teniendo en cuenta un posible papel mediador de la presencia de ira en la muestra de familias estudiadas. La evidencia previa en otros contextos nos hace esperar que un buen funcionamiento familiar se asocie a menores niveles de ira y que esta estructuración a su vez permita predecir mayores niveles de satisfacción con la atención recibida.

\section{Métodos}

\section{Participantes}

Este estudio descriptivo transversal fue llevado a cabo en los servicios sanitarios de urgencias pediátricas de seis centros hospitalarios públicos de la comunidad autónoma de Andalucía, España.

Se propuso la participación, mediante un muestreo no probabilístico por oportunidad, a 874 progenitores cuyos hijos estaban siendo atendidos en esos centros. De ellos sólo 711 estuvieron de acuerdo en participar en el estudio. La muestra final estaba compuesta por 275 padres (38.7\%) y 436 madres (61.3\%), estando sus edades comprendidas entre 18 y 66 años (media $=35.29$, DT $=8.30$ ). Previamente se había hecho un cálculo del tamaño muestral recomendable asumiendo un porcentaje de error del 5\%, un nivel de confianza del $95 \%$, una distribución de repuestas del 50\% y estimando una población pediátrica (menor de 17 años) en las provincias de Andalucía Oriental de 800.000 sujetos. Dicho cálculo indicó una muestra mínima aconsejable de 384 sujetos.

\section{Instrumentos}

Para evaluar el estado de ira se usó la subescala de "Ira como estado" del Inventario de Expresión de Ira Estado-Rasgo-2 (STAXI-2) ${ }^{20}$. Este instrumento es la versión adaptada a la población española del "State-Trait Anger Expression Inventory-2"26. El inventario permite la evaluación de la ira como rasgo y como estado, entre otros índices complejos. La escala de estado usada en este estudio se compone de 15 items los cuales permiten obtener un valor general de ira y la expresión de tres componentes específicos: sentimientos de ira, expresión verbal y expresión física de la misma.

Cada ítem se estructura en un formato de respuesta de cuatro opciones: $1=\mathrm{No}$, en absoluto; $2=$ Algo; $3=$ Moderadamente y $4=$ Mucho. El valor de alpha de Cronbach para la escala de estado de ira en el estudio original de validación de la versión española alcanzó un valor de .89, mientras que en nuestro caso, este valor de consistencia interna fue de .82 .

De cara a determinar la satisfacción, se usó la Escala de Satisfacción con los Servicios Sani$\operatorname{tarios}^{27}$. La escala incluye 11 ítems, cada uno de los cuales admite un rango de respuesta en una escala de 5 puntos que oscila de 1 (no satisfecho en absoluto) a 5 (completamente satisfecho).

La escala se compone de cuatro subescales las cuales determinan el nivel de satisfacción del sujeto con el personal sanitario, con el personal auxiliar, con los elementos del centro de salud y el confort, y con el acceso y el funcionamiento del servicio. Aunque se trata de una escala inicialmente diseñada para ser usada en servicios de atención primaria, dada su estructuración y diseño puede ser usada en servicios de urgencias, como así ha sido en otros estudios ${ }^{17}$. Se trata de una escala corta y fácil de responder que no interfiere con las delicadas circunstancias de la situación de urgencias. Las características psicométricas del instrumento son bastante aceptables con un alpha de Cronbach que oscila entre .80 y .82 para el indicador de expresión de satisfacción general y .59 y .89 para las dimensiones o factores específicos ${ }^{27}$. En nuestro caso el alpha fue de .91 .

Para evaluar el funcionamiento familiar se usó la versión española breve ${ }^{28}$ de la Escala de Cohesión y Adaptabilidad Familiar (FACES II) ${ }^{29,30}$.

Esta versión del FACES II se compone de 20 ítems, que admiten una posibilidad de respuesta estructurada en 5 opciones y que permiten evaluar la percepción de los miembros de una familia sobre la cohesión y adaptabilidad del núcleo familiar en base a dos subescalas, cada una de las cuales se compone por tanto de 10 ítems.

La versión española usada, presenta unas cualidades psicométricas adecuadas como lo demuestra por ejemplo su consistencia interna (alpha de Cronbach: .89 y .87 para las escalas de cohesión y adaptabilidad respectivamente) o su adecuada validez convergente ${ }^{28}$. La consistencia interna en nuestro estudio fue excelente con un alpha de Cronbach que alcanzó 96 para la escala completa, .93 para la escala de cohesión y .91 para la subescala de adaptabilidad.

Adicionalmente se solicitó a cada participante que indicara su edad y su género.

\section{Procedimiento}

En primer lugar se procedió a contactar con las autoridades del Servicio Andaluz de Salud con 
objeto de obtener autorización para conseguir la información en los centros de urgencias hospitalarias participantes de las provincias orientales de Andalucía. Después, en cada centro se solicitó la respectiva aprobación por los comités éticos y de investigación de cara al desarrollo de nuestra investigación.

Los servicios de urgencias pediátricos que participantes en el estudio están ubicados en hospitales infantiles, materno-infantiles o hospitales con plantas y servicios específicos de atención pediátrica. Disponen de una entrada en donde se dispone un box de triaje en el que un pediatra establece un indicador de gravedad de la patología del niño. En virtud de la valoración de la patología el paciente y de la presión asistencial en el momento, éste puede volver a sala de espera para que sea visto siguiendo el orden de llegada, puede pasar a consulta asistida por otro pediatra, o bien, si la sintomatología es importante, puede pasar con urgencia a atención en otra consulta o por otro especialista.

La aplicación de los cuestionarios se llevó a cabo por entrevistadores experimentados, entrenados expresamente para las tareas a desarrollar en la recogida de datos y supervisados por el equipo de investigación para garantizar la homogeneidad y la fiabilidad del proceso de recogida.

Los evaluadores se personaban en los centros y procedían a solicitar la cooperación de las familias después de haber sido atendidas en los departamentos de urgencias pediátricas.

Todas las familias fueron informadas sobre las metas generales de la investigación y, para garantizar la sinceridad, de la naturaleza totalmente anónima de su participación, de la confidencialidad de sus respuestas así como de que sus contestaciones serían tratadas por los investigadores y no serían conocidas por la institución sanitaria. Fueron también informados sobre la posibilidad de abandonar la participación en cualquier momento y se les solicitó la firma de un consentimiento informado. Para conseguir cierta estandarización en los datos, todos los progenitores fueron evaluados por separado en una sala de espera del servicio de urgencias pediátricas o en alguna habitación que facilitara el centro hospitalario, cuando ya había concluido el proceso de atención al menor. Los cuestionarios fueron administrados individualmente.

Los criterios de inclusión fueron que el menor hubiese sido atendido en los servicios de urgencias pediátricas al menos cinco horas antes, voluntariedad en la participación en el estudio (con firma de consentimiento informado) y no haber sido descartado según los criterios de exclusión. Éstos últimos incluían circunstancias específicas que disuadieran, impidieran o desaconsejaran la cumplimentación de los instrumentos de evaluación, tales como la presencia de condiciones en el menor que desaconsejaran la participación de los padres, discapacidad mental o inadecuado nivele educativo general para entender el cuestionario, pobre comprensión del idioma español o no haber firmado el consentimiento informado.

\section{Análisis de datos}

El análisis de datos incluyó un análisis inicial descriptivo y de frecuencias así como la prueba de Shapiro Wilk sobre distribución normal, con objeto de describir la muestra. Los resultados indicaron que los datos no se ajustan a una distribución normal, por lo que en consecuencia, se llevaron a cabo pruebas no paramétricas y ajustadas a esta circunstancia.

Por tanto y de acuerdo a los objetivos planteados, se desarrollaron un análisis de correlaciones bivariadas de Spearman, pruebas de Kruskal Wallis y pruebas de Mann-Whitney para muestras independientes con corrección de Bonferroni. Por último se llevó a cabo un path análisis mediante ecuaciones estructurales. Los análisis fueron llevados a cabo usando la versión española del programa SPSS (versión 11.5) y la versión 6.1 del EQS.

\section{Resultados}

Como se ha comentado, la prueba de normalidad de Shapiro-Wilk indicó que las variables estudiadas en esta investigación no se ajustaban a una distribución normal. Los resultados del test fueron específicamente: satisfacción total $=.99, \mathrm{p}$ $<.00$; cohesión familiar $=.97, \mathrm{p}<.00$; adaptación familiar $=.99, \mathrm{p}<.00$ e ira total - estado $=.99$, $\mathrm{p}<.00$.

Para conocer, de acuerdo con el primero de nuestros objetivos, la asociación entre las diferentes dimensiones de ira consideradas, el funcionamiento familiar y la satisfacción, se llevaron a cabo los correspondientes análisis de correlaciones bivariadas de Spearman, cuyos resultados se detallan en la Tabla 1.

Los resultados muestran en primer lugar que mayores niveles de cohesión y adaptabilidad familiar se asocian a mayores niveles de satisfacción con la atención recibida y que mayores niveles de ira en las dimensiones específicas, se 
Tabla 1. Correlación entre cohesión y adaptabilidad familiar, expresión de ira y satisfacción con la atención recibida.

\begin{tabular}{lccc}
\hline & Adaptabilidad familiar & Cohesión familiar & Satisfacción general \\
\hline Adaptabilidad familiar & & & $0.22^{\star *}$ \\
Cohesión familiar & $.88^{\star *}$ & & $0.18^{\star *}$ \\
Ira - Sentimiento & $-0.12^{\star *}$ & $-0.13^{\star *}$ & $-0.17^{\star *}$ \\
Ira - Expresión física & $-0.10^{\star}$ & $-0.08^{\star}$ & $-0.05(\mathrm{p}=.21)$ \\
Ira - Expresión verbal & $-0.24^{\star *}$ & $-0.25^{\star *}$ & $-0.22^{\star *}$ \\
Ira-Estado General & $-0.17^{\star *}$ & $-0.17^{\star *}$ & $-.20^{\star *}$ \\
\hline
\end{tabular}

* La correlación es significativa al nivel 0,05 (bilateral). ${ }^{* *}$ La correlación es significativa al nivel 0,01 (bilateral).

asocian a menores niveles de satisfacción, excepto en la dimensión de expresión de ira física donde esta relación no es significativa. En la misma línea, mayores niveles de ira durante la atención en urgencias, tanto en las dimensiones específicas como en la expresión general de ira-estado, se asocian a menores niveles de cohesión y adaptabilidad familiar.

En relación con el segundo de nuestros objetivos, nos planteamos llevar a cabo pruebas de Kruskal Wallis y U de Mann-Whitney con corrección de Bonferroni con objeto de confirmar en primer lugar una posible disminución en los niveles de ira en función de la cohesión y la adaptación familiar, en segundo lugar si la satisfacción puede ser mayor conforme los niveles de cohesión y adaptabilidad familiar son mayores y en tercer lugar si la satisfacción es mayor cuando los niveles de ira son menores, respectivamente. Para ello se tuvieron en cuenta los percentiles 25 y 75 y en base a ello, y para cada variable, se consideraron niveles bajos las puntuaciones por debajo del percentil 25, puntuaciones medias aquellas comprendidas entre el percentil 25 y el 75 y se consideraron puntuaciones altas aquellas superiores al valor correspondiente al percentil 75. Con ello se obtuvieron tres grupos de sujetos, uno de nivel bajo, otro medio y otro alto en cada una de las variables incluidas en el análisis Kruskal Wallis y U de Mann-Whitney, es decir, para cohesión familiar, adaptación familiar, los tres indicadores específicos de ira y la variable ira en su expresión general.

Tanto los resultados de la prueba Kruskal Wallis como la U de Mann-Whitney, se detallan en las Tablas 2 y 3 , donde se aprecia que tanto la adaptabilidad familiar, como la cohesión se asocian significativamente a menores niveles de ira y a mayores niveles de satisfacción en los padres. Adicionalmente, niveles menores de ira en las expresiones consideradas se asocian a mayores ni- veles de satisfacción, siendo todas estas relaciones estadísticamente significativas a excepción de la expresión física de ira, que no presentó asociación significativa con la satisfacción general. La expresión de diferencias entre niveles bajos, medios y altos en todas las variables, se detallan en la tabla 3, donde se aprecia generalmente una tendencia no lineal. Como consecuencia de aplicar la corrección de Bonferroni, en las comparaciones entre grupos se adopta como criterio una significatividad asintótica bilateral inferior a 0.017 .

Con objeto de explorar posibles diferencias en ira entre hombres y mujeres en este contexto, se llevó a cabo una prueba no paramétrica como es la U de Man-Withney para muestras independientes. Tanto en Tabla 3 como en la 4, además de los resultados de esta prueba, se incluyen otros estadísticos descriptivos como la media para cada grupo, la desviación típica o el rango promedio utilizado en la prueba de Mann-Whitney.

Los resultados obtenidos indican la inexistencia de diferencia significativa en los niveles de ira, tanto en las dimensiones específicas como en la expresión de ira-estado general, entre padres y madres durante la atención en urgencias.

En relación con el cuarto de nuestros objetivos y para explorar si los dos aspectos del funcionamiento familiar estudiados se asocian a la satisfacción a través de su relación con la ira, se llevó a cabo un Path análisis con el criterio de máxima verosimilitud. Los resultados mostraron un buen ajuste del modelo propuesto a los datos $\left(\mathrm{Chi}^{2}=14.78\right.$ basada en 2 grados de libertad, $\mathrm{p}<$ .00 ; RMSEA $=0.9$; GFI = .99; NNFI = .97; CFI = .99). En la Figura 1 se presentan los parámetros estructurales estandarizados del modelo.

Como se muestra en la Figura 1, la ira es un buen predictor de la satisfacción en los padres, jugando un papel mediador entre el funcionamiento familiar y la satisfacción. Pero, de entre las dos variables analizadas, la cohesión familiar 
Tabla 2. Pruebas de Kruskal Wallis. Ira general en función de la cohesión y adaptabilidad familiar y satisfacción en función de la expresión de ira.

\begin{tabular}{|c|c|c|c|c|}
\hline Variable & Niveles de VI & $\mathbf{N}$ & Rango promedio & $\begin{array}{c}\text { Prueba de Kruskal-Wallis } \\
\text { Chi-cuadrado }(\mathbf{p})\end{array}$ \\
\hline \multirow[t]{3}{*}{ Ira General } & Cohesión familiar baja & 185 & 426.47 & \multirow[t]{3}{*}{$30.44(\mathrm{p}<.00)$} \\
\hline & Cohesión familiar media & 367 & 335.93 & \\
\hline & Cohesión familiar alta & 159 & 320.32 & \\
\hline \multirow[t]{3}{*}{ Ira General } & Adaptabilidad familiar baja & 222 & 421.13 & \multirow[t]{3}{*}{$33.37(\mathrm{p}<.00)$} \\
\hline & Adaptabilidad familiar media & 322 & 321.65 & \\
\hline & Adaptabilidad familiar alta & 167 & 335.66 & \\
\hline \multirow[t]{3}{*}{ Satisfacción general } & Cohesión familiar baja & 185 & 296.15 & \multirow[t]{3}{*}{$28.63(\mathrm{p}<.00)$} \\
\hline & Cohesión familiar media & 367 & 361.02 & \\
\hline & Cohesión familiar alta & 159 & 414.04 & \\
\hline \multirow[t]{3}{*}{ Satisfacción general } & Adaptabilidad familiar baja & 222 & 299.38 & \multirow[t]{3}{*}{$31.32(\mathrm{p}<.00)$} \\
\hline & Adaptabilidad familiar media & 322 & 364.36 & \\
\hline & Adaptabilidad familiar alta & 167 & 415.15 & \\
\hline \multirow[t]{3}{*}{ Satisfacción general } & Ira Sentimiento baja & 246 & 388.77 & \multirow[t]{3}{*}{$26.75(\mathrm{p}<.00)$} \\
\hline & Ira Sentimiento media & 341 & 362.43 & \\
\hline & Ira Sentimiento alta & 124 & 273.32 & \\
\hline \multirow[t]{3}{*}{ Satisfacción general } & Ira Física baja & 362 & 359.46 & \multirow[t]{3}{*}{$5.28(\mathrm{p}<.71)$} \\
\hline & Ira Física media & 217 & 731.71 & \\
\hline & Ira Física alta & 132 & 320.70 & \\
\hline \multirow[t]{3}{*}{ Satisfacción general } & Ira Verbal baja & 315 & 387.43 & \multirow[t]{3}{*}{$50.74(\mathrm{p}<.00)$} \\
\hline & Ira Verbal media & 272 & 373.61 & \\
\hline & Ira Verbal alta & 124 & 237.53 & \\
\hline \multirow[t]{3}{*}{ Satisfacción general } & Ira General baja & 212 & 393.78 & \multirow[t]{3}{*}{$38.54(\mathrm{p}<.00)$} \\
\hline & Ira General media & 362 & 370 & \\
\hline & Ira General alta & 137 & 260.53 & \\
\hline
\end{tabular}

tiene un efecto indirecto significativo sobre la satisfacción, mediatizado por la ira, pero no así la adaptabilidad familiar, cuyo papel no resultó significativo. Y todo ello a pesar de la alta correlación entre cohesión y adaptabilidad familiar (.89; $\mathrm{p}<.00)$. Según la R2, este modelo explica un $5.2 \%$ de la ira total y un $8.1 \%$ de la satisfacción total paterna.

\section{Discusión}

En esta investigación nos planeábamos analizar algunas variables que podrían incidir en el proceso de atención sanitaria en urgencias pediátricas tanto a pacientes como a familiares. Nos interesaba explorar si aspectos inherentes al funcionamiento familiar y a la presencia de alteraciones emocionales, pueden contribuir de manera relevante a la satisfacción de las familias con la atención recibida.

Un primer grupo de objetivos buscaba establecer específicamente asociaciones entre cohe- sión en el funcionamiento familiar y adaptabilidad de las familias en el contexto de urgencias pediátricas, diversas expresiones de ira y la satisfacción que expresan los padres en estos contextos.

Nuestros resultados indican que mayores niveles tanto de cohesión como de adaptabilidad familiar se asocian significativamente a mayores niveles de satisfacción y que cuando los niveles de ira son elevados, los indicadores de funcionamiento familiar analizados, así como la satisfacción en los padres decrecen. Estos resultados confirman lo esperado y van en la línea de los hallazgos de otros estudios que habían encontrado niveles significativos de alteración emocional en contextos sanitarios pediátricos ${ }^{21,22}$. Además la presencia de ira asociada a bajos niveles de satisfacción había sido señalada en un sentido general ${ }^{15}$ profundizando nuestro estudio actual en esta relación y confirmando esta tendencia en manifestaciones específicas de esta variable, tales como ira verbal y como sentimiento. Las circunstancias especiales que se vivencian en urgencias pediátricas hacen que en ocasiones las familias 
Tabla 3. U de Mann-Whitney. Análisis de diferencias en ira y satisfacción general según grupos de niveles de cohesión y adaptabilidad familiar y en función de la expresión de ira.

\begin{tabular}{|c|c|c|c|c|c|c|c|c|}
\hline $\begin{array}{c}\text { Variable } \\
\text { dependiente }\end{array}$ & $\begin{array}{c}\text { Grupos } \\
\text { según la VI }\end{array}$ & $\mathbf{N}$ & Media & Dt & $\begin{array}{c}\text { Rango } \\
\text { promedio }\end{array}$ & $\begin{array}{c}\text { Suma } \\
\text { de Rangos }\end{array}$ & $\begin{array}{c}\text { U de } \\
\text { Mann-Whitney }\end{array}$ & $\begin{array}{l}\text { Sig. Asintótica } \\
\text { (bilateral)* }\end{array}$ \\
\hline \multicolumn{9}{|c|}{ Cohesión Familiar } \\
\hline \multirow[t]{6}{*}{ Ira General } & Nivel bajo & 185 & 21.73 & 5.99 & 323.18 & 59789.00 & 25311.00 & .000 \\
\hline & Nivel medio & 367 & 19.36 & 3.91 & 252.97 & 92839.00 & & \\
\hline & Nivel bajo & & & & 196.28 & 36312.50 & 10307.50 & .000 \\
\hline & Nivel alto & 159 & 18.96 & 3.32 & 144.83 & 23027.50 & & \\
\hline & Nivel medio & & & & 266.97 & 97977.00 & 27904.00 & .423 \\
\hline & Nivel alto & & & & 255.50 & 40624.00 & & \\
\hline \multicolumn{9}{|c|}{ Adaptación Familiar } \\
\hline \multirow[t]{6}{*}{ Ira General } & Nivel bajo & 222 & 21.51 & 5.75 & 316.99 & 70371.50 & 25865.50 & .000 \\
\hline & Nivel medio & 322 & 19.16 & 3.91 & 241.83 & 77868.50 & & \\
\hline & Nivel bajo & & & & 215.64 & 47871.50 & 13955.50 & .000 \\
\hline & Nivel alto & 167 & 19.12 & 3.26 & 167.57 & 27983.50 & & \\
\hline & Nivel medio & & & & 241.32 & 77705.50 & 25702.50 & .421 \\
\hline & Nivel alto & & & & 252.09 & 42099.50 & & \\
\hline \multicolumn{9}{|c|}{ Cohesión Familiar } \\
\hline \multirow[t]{6}{*}{ Satisfacción } & Nivel bajo & 185 & 34.96 & 9.17 & 241.99 & 44769.00 & 27564.00 & .000 \\
\hline & Nivel medio & 367 & 37.41 & 7.38 & 293.89 & 107859.00 & & \\
\hline & Nivel bajo & & & & 147.16 & 27224.50 & 10019.50 & .000 \\
\hline & Nivel alto & 159 & 39.50 & 7.27 & 201.98 & 32115.50 & & \\
\hline & Nivel medio & & & & 251.13 & 92164.00 & 24636.00 & .005 \\
\hline & Nivel alto & & & & 292.06 & 46437.00 & & \\
\hline \multicolumn{9}{|c|}{ Adaptación Familiar } \\
\hline \multirow[t]{6}{*}{ Satisfacción } & Nivel bajo & 222 & 35.32 & 8.18 & 241.70 & 53658.00 & 28905.00 & .000 \\
\hline & Nivel medio & 322 & 37.30 & 7.49 & 293.73 & 94582.00 & & \\
\hline & Nivel bajo & & & & 169.18 & 37558.00 & 12805.00 & .000 \\
\hline & Nivel alto & 167 & 39.69 & 8.10 & 229.32 & 38297.00 & & \\
\hline & Nivel medio & & & & 232.13 & 74744.50 & 22741.50 & .005 \\
\hline & Nivel alto & & & & 269.82 & 45060.50 & & \\
\hline \multicolumn{9}{|c|}{ Ira Sentimiento } \\
\hline \multirow[t]{6}{*}{ Satisfacción } & Nivel bajo & 246 & 38.46 & 7.33 & 307.11 & 75548.00 & 38719.00 & .111 \\
\hline & Nivel medio & 341 & 37.65 & 7.65 & 248.55 & 97030.00 & & \\
\hline & Nivel bajo & & & & 205.16 & 50470.00 & 10415.00 & .000 \\
\hline & Nivel alto & 124 & 33.69 & 9.24 & 146.49 & 18165.00 & & \\
\hline & Nivel medio & & & & 248.88 & 84868.50 & 15726.50 & .000 \\
\hline & Nivel alto & & & & 189.33 & 23476.50 & & \\
\hline \multicolumn{9}{|l|}{ Ira Física } \\
\hline \multirow[t]{6}{*}{ Satisfacción } & Nivel bajo & 362 & 37.48 & 8.02 & 286.31 & 103646.00 & 37943.00 & .493 \\
\hline & Nivel medio & 217 & 37.76 & 7.70 & 296.15 & 64264.00 & & \\
\hline & Nivel bajo & & & & 254.64 & 92180.00 & 21307.00 & .065 \\
\hline & Nivel alto & 132 & 35.71 & 8.33 & 227.92 & 30085.00 & & \\
\hline & Nivel medio & & & & 184.56 & 40049.00 & 12248.00 & .023 \\
\hline & Nivel alto & & & & 159.29 & 21026.00 & & \\
\hline
\end{tabular}

continua

manifiesten estas expresiones de alteración emocional, con un efecto negativo en su satisfacción.

En relación con el funcionamiento familiar, nuestros resultados concuerdan con lo esperado a tenor de otros estudios en contextos sanitarios no de urgencias pediátricas ${ }^{16}$, donde niveles bajos de funcionamiento familiar se asociaban a nive- les elevados de otras alteraciones emocionales diferentes a las consideradas en este estudio. En aquella ocasión era la ansiedad la que mostraba niveles elevados cuando el funcionamiento familiar era deficitario. En nuestro caso teniendo en cuenta la ira y en un contexto de urgencias pediátrico, la asociación es similar. 
Tabla 3. continuación

\begin{tabular}{|c|c|c|c|c|c|c|c|c|}
\hline $\begin{array}{c}\text { Variable } \\
\text { dependiente }\end{array}$ & $\begin{array}{c}\text { Grupos } \\
\text { según la VI }\end{array}$ & $\mathrm{N}$ & Media & Dt & $\begin{array}{c}\text { Rango } \\
\text { promedio }\end{array}$ & $\begin{array}{c}\text { Suma } \\
\text { de Rangos }\end{array}$ & $\begin{array}{c}\text { U de } \\
\text { Mann-Whitney }\end{array}$ & $\begin{array}{c}\text { Sig. Asintótica } \\
\text { (bilateral)* }\end{array}$ \\
\hline \multicolumn{9}{|l|}{ Ira Verbal } \\
\hline \multirow[t]{6}{*}{ Satisfacción } & Nivel bajo & 315 & 38.39 & 7.09 & 299.35 & 94294.50 & 41155.50 & .410 \\
\hline & Nivel medio & 272 & 38.14 & 8.14 & 287.81 & 78283.50 & & \\
\hline & Nivel bajo & & & & 246.08 & 77515.50 & 11314.50 & .000 \\
\hline & Nivel alto & 124 & 32.35 & 8.16 & 153.75 & 19064.50 & & \\
\hline & Nivel medio & & & & 222.31 & 60467.00 & 10389.00 & .000 \\
\hline & Nivel alto & & & & 146.28 & 18139.00 & & \\
\hline \multicolumn{9}{|l|}{ Ira General } \\
\hline \multirow[t]{6}{*}{ Satisfacción } & Nivel bajo & 212 & 38.52 & 7.01 & 300.17 & 63635.00 & 35687.00 & .161 \\
\hline & Nivel medio & 362 & 37.96 & 7.75 & 280.08 & 101390.00 & & \\
\hline & Nivel bajo & & & & 200.12 & 42425.00 & 9197.00 & .000 \\
\hline & Nivel alto & 137 & 33.35 & 8.94 & 136.13 & 18650.00 & & \\
\hline & Nivel medio & & & & 271.42 & 98254.50 & 17042.50 & .000 \\
\hline & Nivel alto & & & & 193.40 & 26495.50 & & \\
\hline
\end{tabular}

* Aplicando la corrección de Bonferroni, se considerará una significatividad asintótica bilateral igual o inferior a 0.017 como punto de corte para considerar la diferencia significativa.

Tabla 4. Diferencia entre hombres y mujeres en la expresión global de ira.

\begin{tabular}{lllll}
\hline \multicolumn{1}{c}{ Variable } & \multicolumn{1}{c}{$\begin{array}{c}\text { Género } \\
\text { Mombres N = 275 } \\
\text { Mujeres N=436 }\end{array}$} & Media (DT) & Rango Promedio & Mann-Whitney \\
\hline Ira-Sentimiento & Hombres & $7.84(2.67)$ & 358.03 & $\mathrm{U}=59390.50$ \\
Ira-Expresión física & Mujeres & $7.74(2.52)$ & 354.72 & $\mathrm{p}=0.83$ \\
& Hombres & $5.92(1.39)$ & 363.69 & $\mathrm{U}=57836$ \\
Ira - Expresión verbal & Mujeres & $5.75(1.06)$ & 351.15 & $\mathrm{p}=0.39$ \\
& Hombres & $6.18(1.61)$ & 349.02 & $\mathrm{U}=58030$ \\
Ira-Estado General & Mujeres & $6.36(1.98)$ & 360.40 & $\mathrm{p}=0.45$ \\
& Hombres & $19.94(4.59)$ & 357.89 & $\mathrm{U}=59430$ \\
& Mujeres & $19.85(4.56)$ & 354.81 & $\mathrm{p}=0.84$ \\
\hline
\end{tabular}

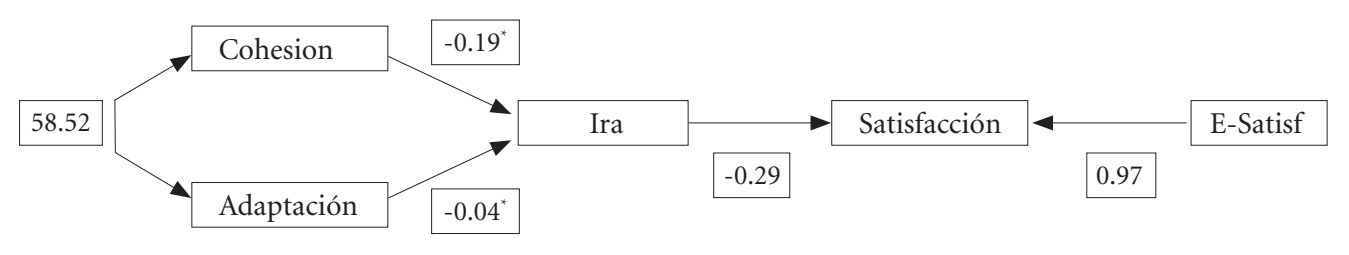

Figura 1. Solución estandarizada del modelo de Path análisis sobre los efectos mediadores de la ira.

El segundo de nuestros objetivos profundiza en estas relaciones confirmando en esta línea que un mejor funcionamiento familiar, donde la cohesión de los miembros de la familia en circunstancias de pérdida de salud y emergencias así como una mejor adaptabilidad a esa situación, puede asociarse a menores niveles de alteración emocional y en concreto ira. Por tanto estas características del funcionamiento familiar harían que a la postre la satisfacción con la atención recibida fuese más satisfactoria. Además, la influencia sobre la vivencia de la experiencia e incluso las connotaciones de cara a la recuperación de la salud o el afrontamiento de las causas de la 
visita a urgencias, mejorarían con un mejor funcionamiento familiar.

La satisfacción parental se ve incrementada cuando los niveles de funcionamiento familiar son adecuados y la ira es reducida, lo que indica una posible interacción entre el funcionamiento familiar y la alteración emocional que quedaría estudiada en el cuarto de nuestros objetivos.

Llama la atención que al ira en su expresión física no presente relaciones significativas con la satisfacción. Este efecto podría deberse a posibles efectos de falta de sinceridad a la hora de cumplimentar el inventario por los participantes y en cualquier caso no coincide con nuestras hipótesis de partida.

Por lo que concierne al tercero de nuestros objetivos, no han sido encontradas diferencias entre padres y madres en sus expresiones de ira en ninguna de las dimensiones específicas consideradas, durante la atención pediátrica. Estos resultados en general confirman lo esperado a excepción de la dimensión física, donde según la bibliografía precedente ${ }^{20}$, los padres podrían haber alcanzado niveles significativamente superiores a las madres. El resultado alcanzado merecería más atención de estudios futuros.

Por último, el cuarto de nuestros objetivos pretendía explorar si el funcionamiento familiar se asocia a niveles diferenciales de satisfacción en los padres, teniendo en cuenta el efecto mediador de la ira, que incidiría en el proceso. Nuestros resultados en general indican que cuanto mayor es la cohesión familiar durante la estancia en urgencias pediátricas, menores son los niveles de ira general en los padres, lo que se asocia a mayores niveles de satisfacción. Por tanto la cohesión familiar tiene efectos indirectos positivos sobre la satisfacción paterna con la atención a sus hijos en urgencias. Sin embargo la adaptabilidad familiar no presenta efectos indirectos significativos a pesar de su fuerte correlación con la cohesión familiar. Esta correlación se explica por tratarse de dos dimensiones bastante relacionada entre sí de un mismo constructo, como es el funcionamiento familiar.

La relación hallada concuerda con los resultados de otros estudios que encontraban que el papel de la cohesión familiar es más importante que la adaptabilidad a la hora de predecir niveles diferenciales de alteraciones emocionales como la ansiedad en procesos de atención de salud como por ejemplo en familias hospitalizadas en servicios de pediatría ${ }^{16}$.

Una explicación plausible de este resultado, se establecería en torno a que la cohesión familiar podría jugar un papel de potenciación del afrontamiento de la alteración emocional en circunstancias y momentos difíciles, con elementos relevantes del funcionamiento familiar como la unidad, el contacto afectivo o la cercanía y la comunicación emocional entre los miembros ${ }^{31,32}$.

Según el análisis de correlaciones, la adaptación familiar mantendría también una relación interesante con la satisfacción, pero no mediatizada a través de la ira.

Nuestros resultados han mostrado un bajo porcentaje de sujetos con niveles de ira medio y alto. Este resultado podría deberse a la posibilidad de que los padres no expresen con el instrumento de evaluación utilizado su nivel real de ira, por un efecto de deseabilidad social o vergüenza con la consiguiente posible alteración de los resultados. Sería interesante que futuros estudios se plantearan la medición de esta variable con otro instrumento o método alternativo.

Como posibles limitaciones del estudio señalaríamos el no haber tenido en cuenta circunstancias específicas que podrían incidir durante el proceso de atención pediátrica en urgencias, tales como la razón o causa de la visita, la gravedad que se otorga a la enfermedad o motivo de la asistencia a urgencias, entre otras variables. La generalización de los resultados ha de ser considerada con reserva teniendo en cuenta que la recogida de datos es relativamente local e igualmente las conclusiones han de ser tomadas con cautela, teniendo en cuenta que se ha usado alguna escala sometida a traducción transcultural. Para estudios futuros sobre estas temáticas podría ser recomendable la inclusión de otros grupos de sujetos para completar el análisis del fenómeno o la toma en consideración de unidades de contexto extrafamiliar, entre otras cuestiones.

No obstante, nuestros resultados otorgan importancia a los procesos inherentes al funcionamiento familiar en la atención sanitaria de urgencias, donde ha de cuidarse la interacción familiar y su funcionamiento. Estos aspectos, no sólo mejorarían los procesos de atención y recuperación de la salud, sino que mejoran el bienestar de todos los protagonistas y la visión que se tiene de los sistemas sanitarios. 


\section{Colaboradores}

A Fernández-Castillo ha colaborado en la concepción y diseño de la investigación, aspectos metodológicos, análisis estadísticos y elaboración de resultados y redacción. MJ Vílchez-Lara ha colaborado en la concepción y diseño de la investigación, aspectos metodológicos, supervisión de la recogida de datos, extracción de conclusiones sobre los resultados y redacción del trabajo.

\section{Reconocimientos}

Este estudio es parte de un proyecto de investigación, sometido a convocatoria y concurrencia pública, subvencionado por la Consejería de Salud del Gobierno Autonómico de la Junta de Andalucía. España. La entidad subvencionadora no ha tomado parte ni a influenciado de ningún modo el diseño del estudio, los análisis de datos, la interpretación de los resultados, la redacción de este trabajo, ni la decisión de someter este estudio a la consideración de esta revista. Los autores han trabajado con total independencia de la entidad que concedió la subvención.

\section{Referencias}

1. Blanco S, Sánchez F, Chinchilla MA, Cobrero EM, Mediavilla M. Satisfacción de los pacientes de urgencias con los cuidados enfermeros. Enferm Clin 2010; 20(1):23-31

2. Fernández-Carbonell MJ, Dasí C, García-Merita ML, Fuentes I. Satisfaction levels of patients and their families with a psychiatric hospital unit. An Psicol 2012; 28(2):378-386.

3. Fernández-Castillo A, Vílchez-Lara MJ, Sada-Lázaro E. Satisfaction with Hospitalization Scale: Adaptation and psychometric properties. Behav Psychol 2012; 20(2):383-400.

4. Kim MK, Blendon RJ, Benson JM. What is driving people's dissatisfaction with their own health care in 17 Latin American countries? Health Expect 2013; 16(2):155-163.

5. Manrique CU, Rivera BS, Barrientos JVR, Esteves CT. Validación de un instrumento para evaluar la satisfacción del usuario durante el periodo preanestésico en el Hospital Regional 1 de Octubre. Rev Esp Med Quir 2012; 17(1):44-50

6. Fernández-Castillo A, Vílchez-Lara M J. Anxiety and parent's beliefs about medication in primary pediatric health care. Clin Sal 2014; 25(2):117-122.

7. Romero-García M, de la Cueva-Ariza L, Jover-Sancho C, Delgado-Hito P, Acosta-Mejuto B, Sola-Ribo M, Juandó-Prats C, Ricart-Basagaña MT, Sola-Sole N. La percepción del paciente crítico sobre los cuidados enfermeros: una aproximación al concepto de satisfacción. Enferm Intensiva 2013; 24(2):51-62.

8. Fernández-Castillo A, Vílchez-Lara MJ, López-Naranjo I. Parental stress and satisfaction during children's hospitalization: differences between immigrant and autochthonous population. Stress Health 2013; 29(1):2230.

9. Soleimanpour H, Gholipouri C, Salarilak S, Raoufi P, Vahidi RG, Rouhi AJ, Ghafouri RR Soleimanpour M. Emergency department patient satisfaction survey in Imam Reza Hospital, Tabriz, Iran. Int J Emerg Med 2011; 4(1):1-7.

10. Stricker KH, Kimberger O, Schmidlin K, Zwahlen M, Mohr U, Rothen HU. Family satisfaction in the intensive care unit: what makes the difference? Intensive Care Med 2009; 35(12):2051-2059.

11. Nager AL, Mahrer NE, Gold JI. State trait anxiety in the emergency department: an analysis of anticipatory and life stressors. Pediatr Emerg Care 2010; 26(12):897-901.

12. Fernández-Castillo A, Vílchez-Lara MJ. Creencias paternas sobre la medicación y satisfacción con la atención sanitaria infantil. Gac Med Mex 2015; 151(3):338344.

13. Fernández-Castillo A, López-Naranjo I. Transmisión de emociones, miedo, y estrés infantil por hospitalización. Int J Clin Health Psychol 2006; 6(3):631-645.

14. Downey LVA, Zun LS. The correlation between patient comprehension of their reason for hospital admission and overall patient satisfaction in the emergency department. J Natl Med Assoc 2010; 102(7):637-643.

15. Fernández-Castillo A, Vílchez-Lara MJ. Factors related to dissatisfaction and anger in parents of children treated at paediatric emergency services. An Pediatr 2015; 82(1):12-18. 
16. Fernández-Castillo A, Vilchez-Lara MJ. Family adaptability, cohesion and anxiety during pediatric hospitalization: Differences between populations of immigrant and non-immigrant origin. J Comp Fam Stud 2011; 42(6):891-901.

17. Xie B, Youash S. The effects of publishing emergency department wait time on patient utilization patterns in a community with two emergency department sites: a retrospective, quasi-experiment design. Int J Emerg Med 2011; 4(1):1-7.

18. Fernández-Castillo A, Vílchez-Lara MJ. Opinión profesional sobre la hospitalización infantil de inmigrantes de origen latinoamericano en Andalucía, España. Rev Salud Publica 2009; 11(3):336-346.

19. Forgays DK, Spielberger CD, Ottaway SA, Forgays DG. Factor structure of the State-Trait Anger Expression Inventory for middle-aged men and women. Assessment 1998; 5(2):141-155.

20. Miguel-Tobal JJ, Casado MI, Cano-Vindel A, Spielberger CD. Manual del inventario de expresión de ira estado-rasgo (STAXI-2). Madrid: TEA Ediciones; 2001.

21. Forrest L, Parker R, Hegarty K, Tuschke H. Patient initiated aggression and violence in Australian general practice. Aust Fam Physician 2010; 39(5):323-326.

22. Zampieron A, Galeazzo M, Turra S, Buja A. Perceived aggression towards nurses: study in two Italian health institutions. J Clin Nurs 2010; 19(15-16):2329-2341.

23. Fernández-Castillo A, Vílchez-Lara MJ. Ansiedad e ira en atención primaria y urgencias pediátricas ¿¿Se enfadan los padres durante la atención pediátrica? Rev Latinoam Psicol 2015; 47(3)205-212.

24. Feldman R. Maternal versus child risk and the development of parent-child and family relationships in five high-risk populations. Dev Psychopathol 2007; 19(02):293-312.
25. Olson DH. Circumplex model of marital and family systems. J Fam Ther 2000; 22(2):144-167.

26. Spielberger CD. Manual for the State-Trait anger expression inventory. Odessa: Psychological Assessment Resources; 1996.

27. Varela J, Rial A, García E. Presentación de una Escala de Satisfacción con los Servicios Sanitarios de Atención Primaria. Psicothema 2003; 15(4):656-661.

28. Martínez-Pampliega A, Iraurgi I, Galíndez E, Sanz M. Family Adaptability and Cohesion Evalutation Scale (FACES): desarrollo de una versión de 20 ítems en español. Int J Clin Hlth Psyc 2006; 6(2):317-338.

29. Olson DH, Portner J, Bell RQ. FACES II. St. Paul: University of Minnesota; 1982.

30. Olson DH, Portner J, Lavee Y. FACES III. St. Paul: University of Minnesota; 1985.

31. Bell CJ, Phillips CR, Haase JE, Monahan PO. Relationship of communication to family adaptability and cohesion in adolescents diagnosed with cancer. J Adolescent Health 2007; 40(2):S29-S29.

32. Locke R, Stefano M, Koster A, Taylor B, Greenspan J. Optimizing patient/caregiver satisfaction through quality of communication in the pediatric emergency department. Pediatr Emerg Care 2011; 27(11):10161021.

Artigo apresentado em 29/05/2015

Aprovado em 21/06/2016

Versão final apresentada em 23/06/2016 